\title{
Self-potential anomalies induced by water injection into hydrocarbon reservoirs
}

\author{
Murtaza Y. Gulamali ${ }^{1}$, Eli Leinov ${ }^{1}$, and Matthew D. Jackson ${ }^{1}$
}

\begin{abstract}
The injection of cold water into a hydrocarbon reservoir containing relatively warmer, more saline formation brine may generate self-potential anomalies as a result of electrokinetic, thermoelectric, and/or electrochemical effects. We have numerically assessed the relative contributions of these effects to the overall self-potential signal generated during oil production in a simple hydrocarbon reservoir model. Our aim was to determine if measurements of self-potential at a production well can be used to detect the movement of water toward the well. The coupling coefficients for the electrochemical and thermoelectric potentials are uncertain, so we considered four different models for them. We also investigated the effect of altering the salinities of the formation and injected brines. We found that the electrokinetic potential peaked at the location of the saturation front (reaching values of $0.2 \mathrm{mV}$ even for the most saline brine considered). Moreover, the value at the production well
\end{abstract}

increased as the front approached the well, exceeding the noise level $(\sim 0.1 \mathrm{mV})$. Thermoelectric effects gave rise to larger potentials in the reservoir $(\sim 10 \mathrm{mV})$, but values at the well were negligible $(\lesssim 0.1 \mathrm{mV})$ until after water breakthrough because of the lag in the temperature front relative to the saturation front. Electrochemical potentials were smaller in magnitude than thermoelectric potentials in the reservoir but were measurable $(>0.1 \mathrm{mV})$ at the well because the salinity front was closely associated with the saturation front. When the formation brine was less saline ( $\sim 1 \mathrm{~mol} /$ liter $)$, electrokinetic effects dominated; at higher salinities ( $\sim 5 \mathrm{~mol} /$ liter $)$, electrochemical effects were significant. We concluded that the measurement of self-potential signals in a production well may be used to monitor the movement of water in hydrocarbon reservoirs during production, but further research is required to understand the thermoelectric and electrochemical coupling coefficients in partially saturated porous media.

\section{INTRODUCTION}

The separation of electrical charge in an electrolyte in response to gradients in pressure, chemical composition, or temperature results in a self-potential (SP) anomaly that maintains overall electroneutrality (e.g., Marshall and Madden, 1959; Corwin and Hoover, 1979; Revil, 1999). In porous media (such as fully or partially saturated rocks), charge separation occurs at the solid-fluid interface when an electrolyte such as brine reacts with the solid surface to leave an excess of (typically) negative charge on the surface and an excess of positive charge in the brine adjacent to the surface (e.g., Wyllie, 1951; Lynch, 1962). This arrangement of charge at the solid-fluid interface is known as the electrical double layer (e.g., Hunter, 1981). The negative charge on the solid surface, known as the Stern layer, is immo- bile, but some of the excess positive charge in the adjacent brine, known as the diffuse layer, is mobile and will move with the fluid. If the brine is subjected to a pressure gradient, it flows relative to the surfaces; some of this positive charge is transported with the flow, giving rise to a streaming current. To balance this current, a conduction current is established. The electrical potential required to maintain this conduction current is the electrokinetic (EK) or streaming potential.

Variations in the chemical composition of the brine result in salinity gradients, down which ions diffuse. However, the mobilities of individual ionic species are different, causing them to migrate at different rates (e.g., Braun and Weingartner, 1985). This effect causes charge separation, which is countered by an electrochemical (EC) potential to maintain electroneutrality (e.g., Revil, 1999). If the surface of the porous medium is not

Manuscript received by the Editor 30 September 2010; revised manuscript received 11 January 2010; published online 16 June 2011.

${ }^{1}$ Imperial College London, Department of Earth Science and Engineering, London, U. K. E-mail: m.gulamali@imperial.ac.uk; e.leinov@imperial.ac.uk; m.d.jackson@imperial.ac.uk.

(C) 2011 Society of Exploration Geophysicists. All rights reserved. 
electrically charged, the EC potential comes solely from the liquid junction or diffusion potential that arises from the mobility contrast between ionic species (e.g., Ortiz et al., 1973).

However, when the surface of the porous medium is electrically charged, an electrical double layer is formed. Consequently, some of the (typically) negative ions in the brine are excluded from the pore space, so a net excess of positive charge migrates down the concentration gradient. This gives rise to a membrane potential (e.g., Revil, 1999). The relative contribution of the membrane and liquid junction potentials to the overall EC potential depends upon the mobility contrast between ionic species and the thickness of the electrical double layer relative to the radius of the brine-occupied pores (e.g., Ortiz et al., 1973). When the porous medium acts as a perfect membrane (i.e., only one species of ion in the brine is transported with the fluid), the membrane potential is the sole contributor to the EC potential.

The thermoelectric (TE) potential has an origin similar to the EC potential. Variations in the temperature of the brine result in a temperature gradient, down which the ionic species migrate. The different mobilities of the different ionic species in the brine cause charge separation, which is countered by the TE potential to maintain electroneutrality (e.g., Revil, 1999).

Several workers have studied SP phenomena during brine injection in geothermal reservoirs (e.g., Corwin and Hoover, 1979; Darnet et al., 2004; Maineult et al., 2006), with the aim of understanding electrical measurements and, ultimately, geothermal activity. In particular, Corwin and Hoover (1979) find SP anomalies ranging from about $50 \mathrm{mV}$ to more than $2 \mathrm{~V}$ in amplitude over distances of $100 \mathrm{~m}$ to $10 \mathrm{~km}$ in a variety of geothermal locations. They suggest that these signals are generated by EK and TE effects resulting from the flow of fluids along faults. Darnet et al. (2004), corrected by Maineult et al. (2006), model the SP signal at the earth's surface when water is injected into a geothermal reservoir. They find that EK effects are dominant when injection rates are larger than 16,000 barrels/day $\left(0.03 \mathrm{~m}^{3} / \mathrm{s}\right)$ but that $\mathrm{TE}$ effects became significant at small injection rates $\left(\lesssim 5000\right.$ barrels/day or $\left.0.01 \mathrm{~m}^{3} / \mathrm{s}\right)$ and after shutin. The EC effects are always negligible. These workers conclude that the interpretation of SP measurements in geothermal areas is not straightforward and requires that EK and TE contributions be considered.

Hydrocarbon production often involves the injection of relatively colder, less saline brine (typically seawater) into a reservoir containing formation brine that is warmer and more saline. Saunders et al. (2006, 2008) and Jaafar et al. (2009b) investigate the behavior of the EK component of the SP generated during oil production in a range of reservoir models. These workers use a 3D finite-element scheme to model multiphase flow and the resulting EK potential in a sandstone reservoir bounded by impermeable conductive shales. They find that the EK signal is a maximum at the water front and decays slowly with distance.

As the moving water front approaches a production well, the EK potential associated with the front encompasses the well even when the front is still some distance away, so the SP measured at the well changes significantly with respect to a distant reference electrode. The EK potential increases with increasing brine salinity because salinity controls the thickness of the electrical double layer and the electrical conductivity of the reservoir rock; as the former decreases and the latter increases, the
EK potential decreases. The EK potential also increases with decreasing reservoir permeability (at fixed production rate) and increasing production rate (for a given reservoir permeability) because it depends upon the pressure gradient into the well. The EK potential is maximized in thick reservoirs with a low shale content because the high electrical conductivity of water-saturated shales bounding the reservoir and/or within the reservoir interval suppresses the signal.

Jaafar et al. (2009b) find that the measured potentials at the well are large enough to be resolved above the backgroundnoise-level $(\sim 0.1 \mathrm{mV})$ in most reservoir and production scenarios. They conclude that the flow of brine toward a production well can be monitored by measuring the EK component of the SP at the well, using electrodes permanently installed downhole, even when the encroaching water front is some distance away $(>100 \mathrm{~m})$ from the well. However, gradients in the brine-phase salinity and temperature may also generate EC and TE potentials. These potentials will contribute to the SP signal measured downhole but are neglected by Saunders et al. $(2006,2008)$ and Jaafar et al. (2009b).

The aim of our study is to extend the work described above and, for the first time and by means of numerical simulation, compare the relative contributions of the EK, EC, and TE potentials to the SP generated in a hydrocarbon reservoir during production. We want to understand how these contributions might vary with respect to the properties of the injected and formation brine and subsequently to determine if the SP measured at a production well can predict fluid flow in a hydrocarbon reservoir.

\section{METHODOLOGY}

\section{Hydrodynamic model}

The ECLIPSE 100 reservoir simulator software (Schlumberger, 2009) is used to solve the equations of multiphase flow in 3D porous media (e.g., Bear, 1972). This fully implicit, threephase, 3D general-purpose black oil simulator uses a finite-difference, finite-volume scheme to solve implicitly for the saturations, pressures, temperatures, and salinities of each fluid phase, given the material properties of the medium and fluids. The software is widely used in the petroleum industry; the advantage for us is that our models are compatible and interchangeable with those used in the industry. We model the flow of a wetting phase (brine, subscript $w$ ) and an immiscible nonwetting phase (oil, subscript $o$ ) through a hydrocarbon reservoir. We neglect the cross coupling to the electrodynamic problem (e.g., Fitterman, 1978); we also assume that there is rapid heat transfer between the fluid phases and the porous medium (e.g., Hassanizadeh and Gray, 1993) and that the transport of salt and heat is dominated by advection (e.g., Bear, 1972). This assumption is reasonable for the modeled flow rates where advective processes dominate over diffusive processes.

The relative permeability of each phase is described as

$$
\begin{gathered}
k_{\mathrm{r} w}=k_{w}^{\prime} S_{w n}^{\lambda}, \\
k_{\mathrm{r} o}=k_{o}^{\prime}\left(1-S_{w n}\right)^{\lambda},
\end{gathered}
$$

where the primed variables are the corresponding maximum relative permeabilities, $\lambda$ is an exponent related to the pore-size distribution of the medium (e.g., Bear, 1972), and 


$$
S_{w n}=\frac{S_{w}-S_{w c}}{1-S_{w c}-S_{o r}}
$$

is the normalized brine saturation. Here, $S_{w}$ is brine saturation, $S_{w c}$ is connate water saturation, and $S_{o r}$ is residual oil saturation.

Our 3D model (Figure 1) is based on that of Saunders et al. (2008) and is discretized on a regular hexahedral mesh that is refined around the production well to allow us to determine the flow variables accurately there. It consists of a sandstone reservoir saturated with oil and formation brine. The initial (formation) brine saturation is set to $S_{w}=S_{w c}=0.2$, and the oil saturation is set to $S_{o}=1-S_{w c}=0.8$. A relatively colder, less saline brine is injected at the left-hand face of the reservoir at a rate of 10,000 barrels/day $\left(\sim 1.84 \times 10^{-2} \mathrm{~m}^{3} / \mathrm{s}\right)$ so that $S_{w}=1-S_{o r}=0.8$ and $S_{o}=S_{o r}=0.2$ at this boundary. Oil, and later brine, are produced at a single vertical production well located $1 \mathrm{~km}$ from this inlet boundary. Setting $k_{w}^{\prime}=0.3$, $k_{o}^{\prime}=0.8, \lambda=4$, the fluid phase viscosities to $\mu_{w}=\mu_{o}=10^{-3}$ $\mathrm{Pa} \cdot \mathrm{s}$, and the fluid densities to $\rho_{w}=\rho_{o}=10^{3} \mathrm{~kg} / \mathrm{m}^{3}$ yields a shock-front-dominated displacement of oil by brine with neither gravity nor capillary effects. This is typical of production in many water-wet sandstone reservoirs, particularly near a production well where viscous forces dominate (e.g., Dake, 1978; Anderson, 1987).

\section{Electrodynamic model}

The electrodynamic problem is coupled to the hydrodynamic problem via (e.g., Revil, 1999)

$$
\mathbf{j}=-\sigma_{\mathrm{fs}} \nabla U+L_{\mathrm{EK}} \nabla\left(P_{w}-\rho_{w} g z\right)+L_{\mathrm{TE}} \nabla T+L_{\mathrm{EC}} \nabla C_{f},
$$

where $\mathbf{j}$ is the electric current $(\mathrm{A}) ; \sigma_{\mathrm{fs}}$ is the electrical conductivity of the partially saturated porous medium $(\mathrm{S} / \mathrm{m}) ; U$ is the self-potential of the brine $(\mathrm{V}) ; L_{\mathrm{EK}}, L_{\mathrm{TE}}$, and $L_{\mathrm{EC}}$ are coupling terms for the fluid potential, temperature, and salinity, respectively; $P_{w}$ is the brine pressure $(\mathrm{Pa}) ; g$ is gravitational acceleration $\left(9.821 \mathrm{~m} / \mathrm{s}^{2}\right) ; T$ is the temperature of the fluid phases and the porous medium $(\mathrm{K})$; and $C_{f}$ is the salinity of the brine phase (mol/liter).

The coupling terms are described in more detail in the following subsection. The electrical conductivity of the partially saturated porous medium is calculated using Archie's law (e.g., Telford et al., 1990):

$$
\sigma_{\mathrm{fs}}=\phi^{m} \sigma_{w} S_{w}^{n}
$$

where $\phi$ is the porosity of the porous medium, $m$ is the cementation exponent of the rock, $n$ is the saturation exponent, and $\sigma_{w}$ a)

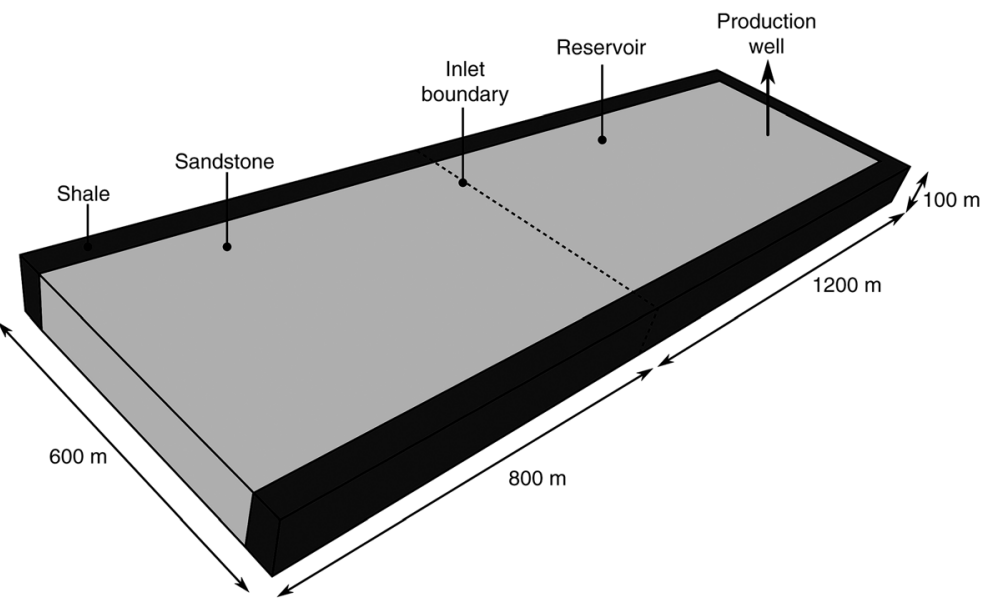

b)

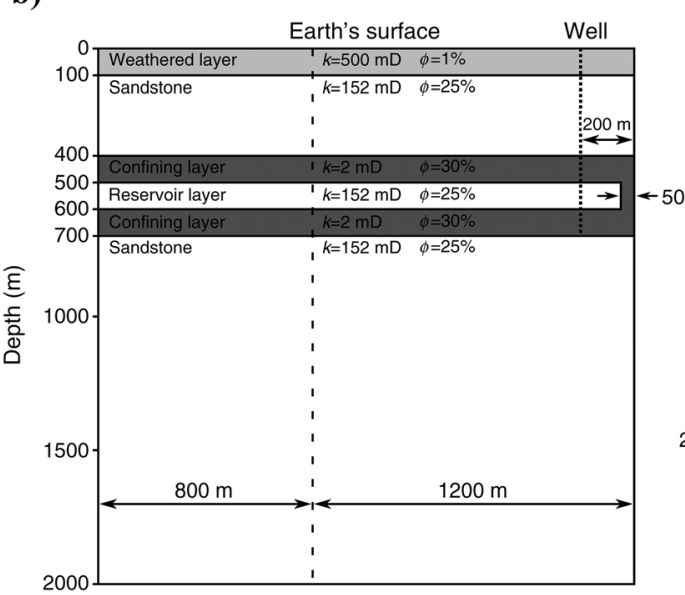

c)

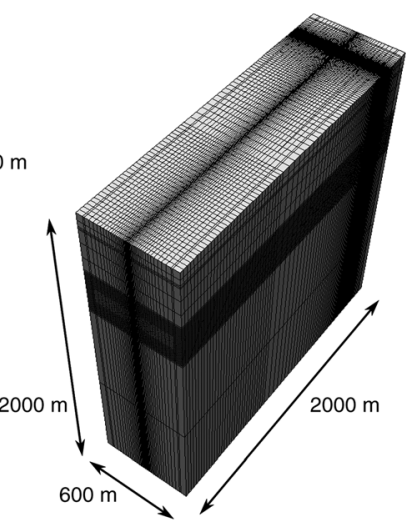

Figure 1. (a) The reservoir layer consists of a $1150 \times 500 \times 100-\mathrm{m}$ sandstone reservoir bounded on three of its four vertical sides by 50 -m-wide shales with a relatively higher porosity and lower permeability. The fourth side is bounded by 800 $\mathrm{m}$ of sandstone with the same material properties as the reservoir and the same fluid saturation as the inlet boundary. Brine is injected at this boundary, and oil is produced at the production well. (b) Vertical cross section through our entire geologic model, based on Saunders et al. (2008). The reservoir layer lies between 500 and $600 \mathrm{~m}$ depth between two impermeable shale layers. The location of the production well is indicated by the dotted vertical line. The injection boundary is marked by the dashed vertical line. (c) A 3D finite-element representation of our geologic model. The computational domain is refined in the reservoir layer, particularly around the well. 
is the electrical conductivity of the brine $(\mathrm{S} / \mathrm{m})$, which is related to the brine salinity by (Worthington et al., 1990)

$$
\begin{aligned}
\log _{10} C_{f}=- & 1.03024+1.06627\left(\log _{10} \sigma_{w}\right) \\
+ & 2.41239 \times 10^{-2}\left(\log _{10} \sigma_{w}\right)^{2} \\
+ & 3.68102 \times 10^{-3}\left(\log _{10} \sigma_{w}\right)^{3} \\
+ & 1.46369 \times 10^{-4}\left(\log _{10} \sigma_{w}\right)^{4}
\end{aligned}
$$

We set $m=1.8$ and $n=2$; these values are typical of shallow, water-wet, consolidated sandstone (e.g., Freedman and Ausburn [1985] and references therein; Anderson [1986]).

Following the method of Saunders et al. (2008), our reservoir model is extended by introducing 50-m-wide shales, with a relatively higher porosity and lower permeability, around three of the four vertical sides of the reservoir; the fourth side is bounded with 800 -m-wide sandstone (Figure 1a). The reservoir is bounded above and below by 100-m-thick shales, and these in turn are bounded by further sandstone layers and a weathered layer at the earth's surface (Figure 1b). These additional parts form a geologic model that allows us to use the electrical potential at the outer boundary as a reference potential (e.g., Saunders et al., 2008); numerical tests show that increasing the size of the model domain beyond that used does not significantly affect the results. In reality, the reference would be one or more distant electrodes. The entire geologic model simulates a faultbounded hydrocarbon reservoir wherein the injection of brine from a distant injection well displaces oil toward the production well.

We assume there are no external current sources or sinks and the net current flow through the boundaries of the model is zero, i.e.,

$$
\nabla \cdot \mathbf{j}=0
$$

Furthermore, we set $U=0$ at all boundaries except the lefthand face, where we set $\nabla U=0$. At the earth's surface, we set $\mathbf{j} \cdot \widehat{\mathbf{n}}=0$, where $\widehat{\mathbf{n}}$ is the unit vector normal to the earth's surface. We also set $\nabla\left(P_{w}-\rho_{w} g z\right)=\nabla T=\nabla C_{f}=0$ on all boundaries of our model. The electrodynamic problem is solved using the finite-element scheme described by Saunders et al. (2008), except that we include the additional terms in equation 4 to describe EC and TE effects, which were neglected in the earlier study. The ECLIPSE reservoir simulator determines the values of the hydrodynamic variables at the center of each of the elements in the reservoir mesh, so we extrapolate the brine-phase potential, temperature, and salinity to each of the nodes in the mesh by setting these variables at each node to be the volume-averaged value of the elements sharing that node.

\section{Electrical coupling terms}

The coupling terms $L_{\mathrm{EK}}, L_{\mathrm{TE}}$, and $L_{\mathrm{EC}}$ can be described in terms of the coupling coefficients, $C_{\mathrm{EK}}(\mathrm{V} / \mathrm{Pa}), C_{\mathrm{TE}}(\mathrm{V} / \mathrm{K})$, and $C_{\mathrm{EC}}(\mathrm{V} \cdot$ liter $/ \mathrm{mol})$ :

$$
\begin{aligned}
& L_{\mathrm{EK}}=\sigma_{\mathrm{fs}} C_{\mathrm{EK}}, \\
& L_{\mathrm{TE}}=\sigma_{\mathrm{fs}} C_{\mathrm{TE}}, \\
& L_{\mathrm{EC}}=\sigma_{\mathrm{fs}} C_{\mathrm{EC}} .
\end{aligned}
$$

We express each coefficient in terms of a factor that is dependent upon the brine salinity and temperature and a factor known as the relative coupling coefficient, which is dependent upon the brine saturation and varies between zero and one (e.g., Jackson, 2008):

$$
\begin{gathered}
C_{\mathrm{EK}}=C_{\mathrm{EK} \mid S_{w}=1} C_{r, \mathrm{EK}}, \\
C_{\mathrm{TE}}=C_{\mathrm{TE}}^{p} C_{r, \mathrm{TE}}, \\
C_{\mathrm{EC}}=C_{\mathrm{EC}}^{p} C_{r, \mathrm{EC}},
\end{gathered}
$$

where the terms subscripted $r$ are the relative coupling coefficients and where $C_{\mathrm{EK} \mid S_{w}=1}, C_{\mathrm{TE}}^{p}$, and $C_{\mathrm{EC}}^{p}$ are the values of the EK, TE, and EC coupling coefficients when $S_{w}=1$.

For sandstone rocks, $C_{\mathrm{EK} \mid S_{w}=1}$ is strongly dependent upon brine salinity. Jaafar et al. (2009) and Vinogradov et al. (2010) show that as the salinity of the brine increases, the excess countercharge in the diffuse part of the electrical double layer in water-wet sandstones is reduced, thus decreasing the magnitude of the coupling coefficient. We describe the salinity dependence of $C_{\mathrm{EK} \mid S_{w}=1}$ using the following expression, obtained from a linear least-squares fit of the measured data of Jaafar et al. (2009) at $25^{\circ} \mathrm{C}$ :

$$
C_{\mathrm{EK} \mid S_{w}=1}=-10^{-6} \times C_{f}^{-1.213},
$$

in millivolts/pascal. The coefficient of determination of this fit is $R^{2}=0.998$.

The EK relative coupling coefficient is a function of brine saturation because it is dependent upon the excess countercharge in the diffuse layer; the countercharge is transported by the flow of brine (e.g., Jackson, 2010). It can be expressed in many ways (see Jackson [2010] for a review), but we use the simple form suggested by Saunders et al. (2008):

$$
C_{\mathrm{r}, \mathrm{EK}}=S_{w n}^{h},
$$

with $h=0.4$ because this agrees with the results of Jackson (2010) for water-wet capillaries occupied by water and a nonwetting, nonpolar oil phase. Saunders et al. (2008) find that the EK potential at the production well is not sensitive to the value of this exponent for the type of shock-front-dominated displacement modeled here.

Few data are available for the TE and EC coupling coefficients, particularly during multiphase flow. We assume that the saturation dependence of the coupling coefficients is captured by allowing them to vary between the limiting cases described in the Introduction, when the porous medium acts as a perfect membrane (superscript $p$ ) and when the porous medium is uncharged (superscript $u$ ).

Leinov et al. (2010) measure the TE coupling coefficient in fully saturated intact sandstone samples for a range of sodium chloride $(\mathrm{NaCl})$ brine salinities, accounting for temperature-dependent electrode effects. They find that at low salinity $\left(10^{-4}\right.$ $\mathrm{mol} /$ liter), $C_{\mathrm{TE}}$ exhibits the salinity dependence predicted for a perfect membrane; at high salinity ( $1 \mathrm{~mol} / \mathrm{liter})$, the coupling coefficient follows that predicted for an uncharged porous medium. They explain their results by noting the salinity dependence of the thickness of the electrical double layer. At low salinity, the electrical double layer is thicker, so co-ions are excluded from the pore space; at high salinity, the electrical double layer is thinner and has little impact on charge transport. 
We use their model for the limits of the TE coupling coefficient, which explicitly accounts for salinity dependence:

$$
\begin{gathered}
C_{\mathrm{TE}}^{p}=-1.984 \times 10^{-1}\left(\log _{10} C_{f}\right) \\
+5.953 \times 10^{-1}, \\
C_{\mathrm{TE}}^{u}=-1.984 \times 10^{-1}\left(2 t_{\mathrm{Na}}-1\right)\left(\log _{10} C_{f}\right) \\
+1.059 t_{\mathrm{Na}}-5.673 \times 10^{-1},
\end{gathered}
$$

in millivolts/Kelvin, where $t_{\mathrm{Na}}$ is the Hittorf transport number for sodium ions:

$$
t_{\mathrm{Na}}=\left\{\begin{array}{lc}
0.39, & C_{f}<0.09 \\
3.66 \times 10^{-1} & C_{f} \geq 0.09 \\
-2.12 \times 10^{-2}\left(\log _{10} C_{f}\right) &
\end{array}\right.
$$

with $C_{f}$ in moles/liter.

The EC coupling coefficient is modeled in charged and uncharged granular porous material saturated with a binary symmetric 1:1 electrolyte by Revil (1999). His model agrees with published experimental data for shaly sands (see references in Revil [1999]). We use the predicted limits of $C_{\mathrm{EC}}$ for an $\mathrm{NaCl}$ brine:

$$
\begin{gathered}
C_{\mathrm{EC}}^{p}=-8.61 \times 10^{-2} \frac{T}{C_{f}}, \\
C_{\mathrm{EC}}^{u}=2.07 \times 10^{-2} \frac{T}{C_{f}}
\end{gathered}
$$

(in $\mathrm{mV}$. liters $/ \mathrm{mol}$ ). The relative coupling coefficients $C_{\mathrm{r}, \mathrm{TE}}$ and $C_{\mathrm{r}, \mathrm{EC}}$ vary as a function of brine saturation because, as with $C_{\mathrm{r}, \mathrm{EK}}$, they depend upon the volume of water occupying the pore space of the rock.

We investigate several different relationships between brine saturation and salinity ahead of, and behind, the displacing brine front. First, we assume that if the wetting brine saturation is low or if the brine salinity is low, then the brine is located entirely within the electrical double layer, excluding co-ions and causing the rock to behave as a perfect membrane. Second, we assume that if the brine saturation is high or the salinity is high, the porous medium behaves as if it is uncharged. If the brine saturation is low and the salinity is high or vice versa, then the behavior may be more complex, so we investigate end-member cases in which the rock behaves as a perfect membrane or as an uncharged porous medium.

In the absence of any data or model to describe the saturation dependence of the TE and EC relative coupling coefficients, we assume a simple linear relationship with brine saturation within the following limits:

- Case A - A perfect membrane ahead of the brine saturation front where the saturation is low and a perfect membrane behind the front where the brine salinity is low.

- Case B - A perfect membrane ahead of the front where the brine saturation is low and an uncharged porous medium behind the front where the saturation is high.

- Case C - An uncharged porous medium ahead of the front where the brine salinity is high and a perfect membrane behind the front where the salinity is low.

- Case D - An uncharged porous medium ahead of the front where the brine salinity is high and an uncharged porous medium behind the front where the brine saturation is high.
These four limits or cases can be modeled as

$$
\begin{gathered}
C_{r, \mathrm{X}}=1, \\
C_{r, \mathrm{X}}=1+S_{w n}(\alpha-1), \\
C_{r, \mathrm{X}}=\alpha+S_{w n}(1-\alpha),
\end{gathered}
$$

and

$$
C_{r, \mathrm{X}}=\alpha,
$$

where $C_{r, \mathrm{X}}$ is $C_{r, \mathrm{TE}}$ or $C_{r, \mathrm{EC}}$ and where $\alpha$ is $C_{\mathrm{TE}}^{u} / C_{\mathrm{TE}}^{p}$ or $C_{\mathrm{EC}}^{u} / C_{\mathrm{EC}}^{p}$, respectively. Equations $15 \mathrm{a}-15 \mathrm{~d}$ correspond to cases A-D, respectively, and are illustrated in Figure 2, where we show the relative coupling coefficient as a function of brine saturation.

\section{RESULTS AND DISCUSSION}

\section{SP anomalies induced by water injection}

We begin by simulating oil production by injecting $0.5-\mathrm{mol} /$ liter brine at $30^{\circ} \mathrm{C}$ into a reservoir containing 5-mol/liter formation brine at $80^{\circ} \mathrm{C}$. This represents the injection of seawater into a reservoir containing very saline formation brine, for which the EK potential will be small (as discussed by Saunders et al., 2008). Figure 3 shows the profile along a line through the center of the reservoir, from the inlet boundary toward the well, for four different time steps in the simulation until water breakthrough occurs. For clarity, only cases $\mathrm{A}$ and $\mathrm{D}$ of $C_{\mathrm{TE}}$ and $C_{\mathrm{EC}}$ are shown because these represent the end members. All four cases are illustrated in Figure 4.

As the brine saturation front moves toward the well, so also do the temperature and salinity fronts. The peak in the EK potential is associated with the saturation front (Saunders et al., 2008), so the value of the EK potential at the well increases in magnitude as the front approaches, resulting in a potential of approximately $0.2 \mathrm{mV}$ when water breaks through at the well. The nonzero value of the EK potential at the inlet boundary $(\sim-0.7 \mathrm{mV})$ arises because the boundary is a current source;

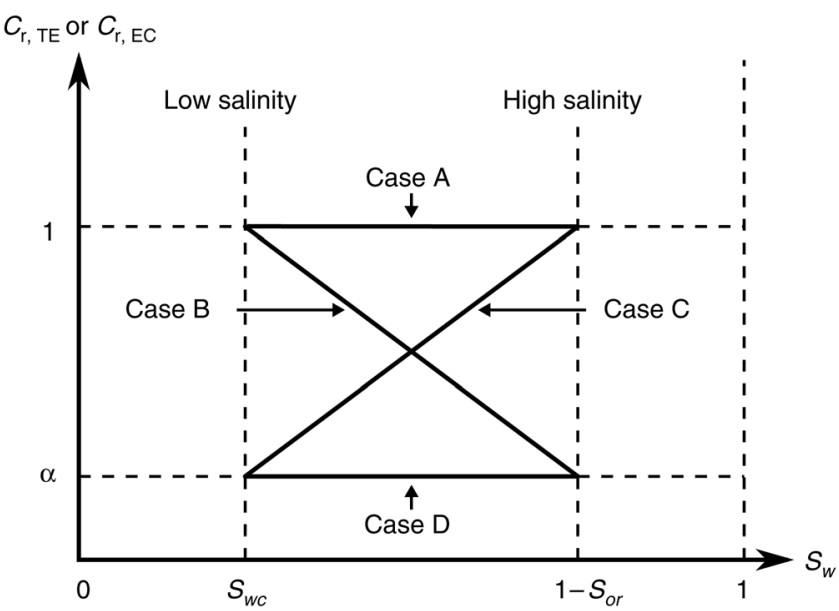

Figure 2. Four possible models of the TE or EC relative coupling coefficient as a function of the brine saturation, labeled as cases A-D as described in the text. 
the potential falls back to zero at the left-hand far-field boundary of the model (1800 $\mathrm{m}$ from the well; not shown).

The magnitude of the TE potential is significantly greater than the EK potential for the four time steps shown in Figure 3. This is expected because the magnitude of the TE coupling coefficient, for both cases of $C_{\mathrm{TE}}$ shown here, is greater than the magnitude of the EK coupling coefficient for the range of salinities considered. However, the change in TE potential occurs at the temperature front, so the lag in the temperature front relative to the saturation front causes the TE potential to be relatively small $(<0.2 \mathrm{mV})$ at the well, even after water breakthrough occurs.

The magnitude of the EC potential is greater than the EK and TE potentials for the four time steps shown in Figure 3. Moreover, the change in EC potential occurs at the salinity front and, because the salinity front more closely follows the saturation front, the $\mathrm{EC}$ signal for the cases of $C_{\mathrm{EC}}$ shown here is larger than the TE signal at the well $(\gtrsim 1 \mathrm{mV})$ when water breakthrough occurs.

In Figure 4, we present simulated values at the center of the production well as a function of time until water breakthrough occurs. The EK potential at the well begins to rise about 400 days $(100 \mathrm{~m})$ before the brine reaches the well and is $\gtrsim 0.1 \mathrm{mV}$
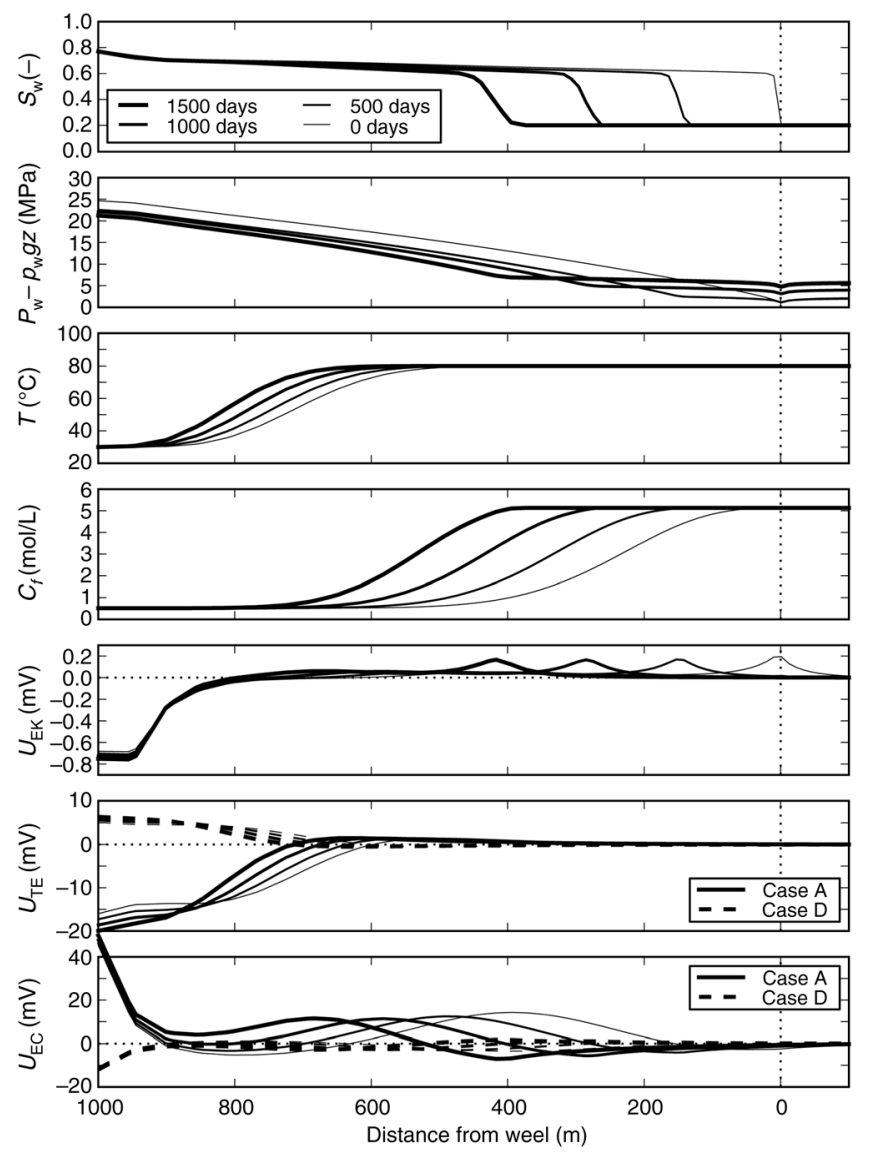

Figure 3. (Top to bottom) Brine saturation, fluid potential, temperature, salinity, EK potential, TE potential, and EC potential as a function of distance from the production well (marked by the vertical dotted line) along a 1D horizontal section through the center of the model at four different times (denoted by lines of different thickness) until water breakthrough occurs. approximately 100 days before breakthrough occurs. If we consider $\pm 0.1 \mathrm{mV}$ to be the conservative limit of an SP signal that may be measured in the subsurface (Chen et al., 2006), this finding agrees with Saunders et al. (2008) and suggests that EK potentials can be resolved in the subsurface, making them useful for detecting water encroaching on a well some time (and distance) before breakthrough occurs, even for very saline formation brines.

The TE potential for cases A and $\mathrm{B}$ of $C_{r, \mathrm{TE}}$ is the same; likewise is the potential for cases $\mathrm{C}$ and $\mathrm{D}$ of $C_{r, \mathrm{TE}}$. The common aspect of each pair of cases is the nature of the relative coupling coefficient ahead of the brine saturation front, suggesting that the TE potential is more sensitive to the value of $C_{r \text {.TE }}$ ahead of the saturation front than behind it. The magnitude of the TE potential for all cases of $C_{\mathrm{r}, \mathrm{TE}}$ increases with time until water breakthrough occurs. However, in comparison to the EK potential, it does not rise as quickly. Moreover, for cases C and D, the TE potential never becomes greater than $0.05 \mathrm{mV}$, even after water breakthrough. This implies that the TE component of the SP measured at the production well in a hydrocarbon reservoir is negligible relative to the other components. Maineult et al. (2006) find that the TE potential contributes about $10 \%$ of the overall SP signal in their model for the same injection rate as ours, but they consider a geothermal reservoir where the
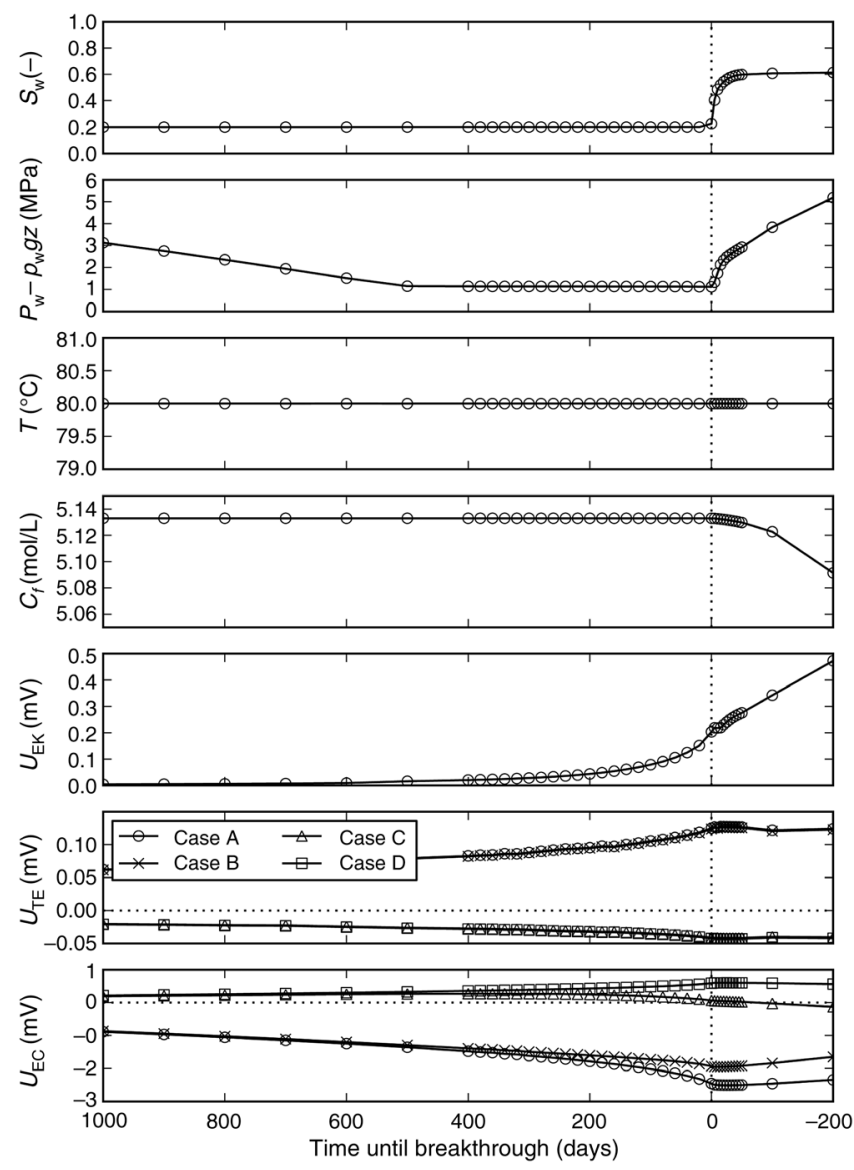

Figure 4. (Top to bottom) Brine saturation, fluid potential, temperature, salinity, EK potential, TE potential, and EC potential as a function of time until water breakthrough occurs at the production well, at the center of the borehole. 
temperature difference between the injected and in situ water is approximately $140^{\circ} \mathrm{C}$.

The magnitude of the EC potential for all cases of $C_{r, \mathrm{EC}}$ is already $\gtrsim 0.2 \mathrm{mV} 1000$ days before water breakthrough occurs. For cases $\mathrm{A}, \mathrm{B}$, and $\mathrm{D}$ of $C_{r, \mathrm{EC}}$, the magnitude of the $\mathrm{EC}$ potential increases as the saturation front approaches the well, indicating that it would be measurable in the subsurface and would contribute more to the overall SP signal than the EK or TE potentials for the relatively large salinity contrast between the formation and injected brine and a very saline formation brine, considered here. These results are contrary to Maineult et al. (2006), who find the EC potential to be negligible in geothermal

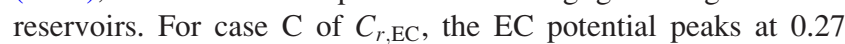
$\mathrm{mV}$, approximately 350 days before water breakthrough occurs, and thereafter decreases to $0.07 \mathrm{mV}$ at breakthrough.

The shape of the EC potential for case $\mathrm{B}$ of $C_{r, \mathrm{EC}}$ follows the shape of case $\mathrm{A}$, whereas the shape of the potential for case $\mathrm{C}$ of $C_{r, \mathrm{EC}}$ follows the shape of case $\mathrm{B}$. This implies that the EC potential is more sensitive to the value of $C_{r, \mathrm{EC}}$ ahead of the saturation front than behind it; similar behavior is observed for the TE potential.

Figure 5 shows the magnitude of the change in the total SP, $\left|\Delta U_{\text {total }}\right|$, relative to the SP at the well 1000 days before breakthrough, at the center of the production well as a function of time until water breakthrough occurs for each case of the relative coupling coefficient. For cases A, B, and D of the relative coupling coefficient, $\left|\Delta U_{\text {total }}\right|$ increases as the saturation front approaches the well and is above the expected level of background noise $(0.1 \mathrm{mV})$ marked by the dashed horizontal line on Figure 5 at least 600 days before breakthrough. These results suggest that changes in SP in high-salinity formations will be measurable downhole at least 2 years before water breakthrough and, moreover, could be used to monitor water approaching the well. The value of $\left|\Delta U_{\text {total }}\right|$ for case $\mathrm{C}$ of the relative coupling coefficient is $0.1 \mathrm{mV}$ until sometime after breakthrough, suggesting that, for this case, no change in the SP would be measured in the subsurface as water approached the production well.

The relative contributions of the change in each of the SP signals at the center of the production well, as a function of time

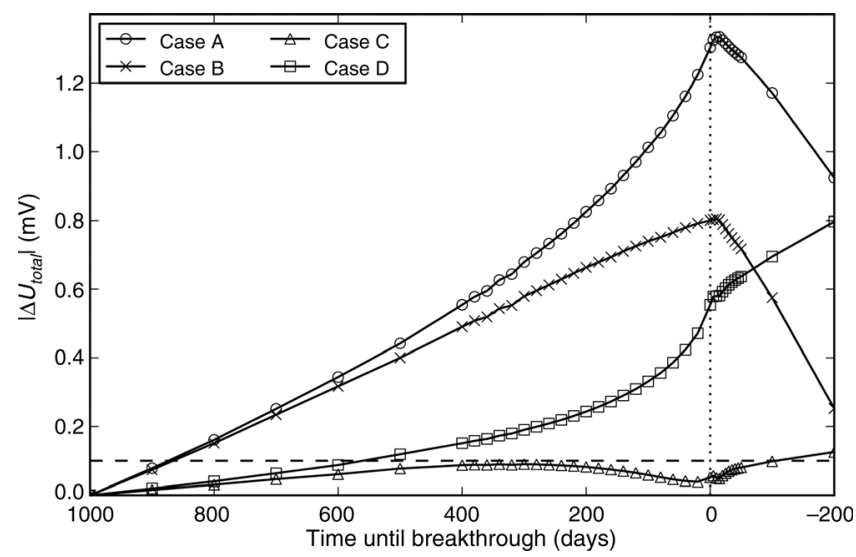

Figure 5. Magnitude of the change in the TE potential relative to the potential 1000 days before breakthrough as a function of time until water breakthrough occurs at the production well, at the center of the borehole for each case of the relative coupling coefficient. until water breakthrough occurs for each case of the relative coupling coefficient, is compared in Figure 6. Initially for all cases of the relative coupling coefficient, the contribution of the EC potential dominates the EK and TE potentials, and the contribution of the TE potential is greater than the EK potential. However, as the saturation front approaches the well, the contribution of the EK potential grows and the contribution of the EC potential falls. In particular, for case $\mathrm{C}$ of the relative coupling coefficient, the contribution of the EK potential is greater than the contribution of the EC potential approximately 200 days before breakthrough. This behavior reduces the SP for case $\mathrm{C}$ observed in Figure 5.

The change in the TE potential for cases A, B, and D of the relative coupling coefficient contributes to $\lesssim 5 \%$ of the change in SP at the well. For case $\mathrm{C}$ of the relative coupling coefficient, the change in the TE potential contributes to as much as $14 \%$ of the change in SP approximately 100 days before breakthrough. However, the contribution from the change in the EC potential falls at this time, and the contribution from the change in the EK potential peaks at about $80 \%$.

The results suggest that the EK and EC potentials may have a notable contribution to the SP signal measured at the production well in a hydrocarbon reservoir. Although the TE potential in the reservoir may be relatively large, its value at the well will be small because the temperature front lags behind the saturation front.

\section{Effect of formation brine salinity}

We now consider the sensitivity of the EK and EC components of the SP signal to the salinity of the formation brine. Following the results of the previous subsection, we neglect the TE potential because it did not significantly contribute to the SP at the well. Moreover, the TE coupling coefficient is weakly sensitive to salinity changes, varying by $\lesssim 0.1 \mathrm{mV} \cdot \mathrm{mol} /$ liter over the range of salinities considered here. As before, we simulate oil production by injecting $0.5-\mathrm{mol} /$ liter brine at $30^{\circ} \mathrm{C}$ into a reservoir containing formation brine at $80^{\circ} \mathrm{C}$.

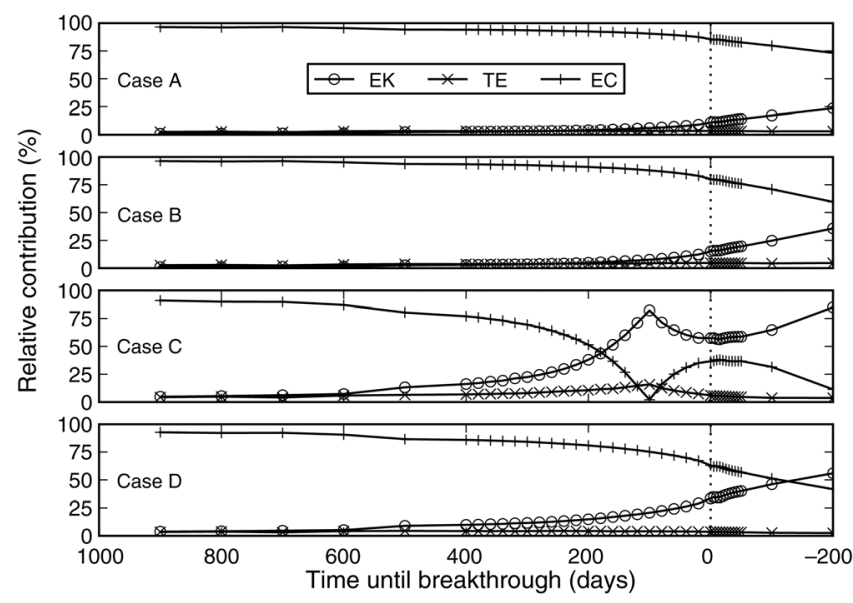

Figure 6. Relative contribution of the change in the EK, TE, and EC potentials to the change in the overall SP signal as a function of time until water breakthrough occurs at the well, at the center of the borehole, for each case of the relative coupling coefficient. 
Figure 7 shows the EK potential, EC potential for each case of $C_{r, \mathrm{EC}}$, and change in the SP signal for each case of $C_{r, \mathrm{EC}}$ as a function of time until water breakthrough occurs at the production well, at the center of the borehole, for different formationbrine salinities. As expected, the EK potential increases as the salinity of the formation brine decreases (Jaafar et al., 2009a; Vinogradov et al., 2010). The limits of the EC coupling coefficient are inversely proportional to $C_{f}$, but the magnitude of the EC potential decreases as the salinity of the formation brine decreases because the salinity gradient $\nabla C_{f}$ is considerably less at lower salinities. It is unlikely that the EC contribution is observable in the subsurface at lower salinity.

Examining the SP for case A of the relative coupling coefficient in Figure 7, the magnitude of the potential remains above $0.1 \mathrm{mV}$ prior to water breakthrough for all formation brine salinities except 1-mol/liter. For this salinity, the SP signal remains between roughly -0.1 and $0.1 \mathrm{mV}$ until about 150 days before water breakthrough occurs. Thereafter, it rises to $1.2 \mathrm{mV}$ at breakthrough. The SP for case B of the relative coupling coefficient behaves in the same way as case A, with the potential for the 1-mol/liter salinity formation brine rising above $0.1 \mathrm{mV}$ approximately 200 days before breakthrough. The magnitude of the SP for the 2-mol/liter-salinity formation
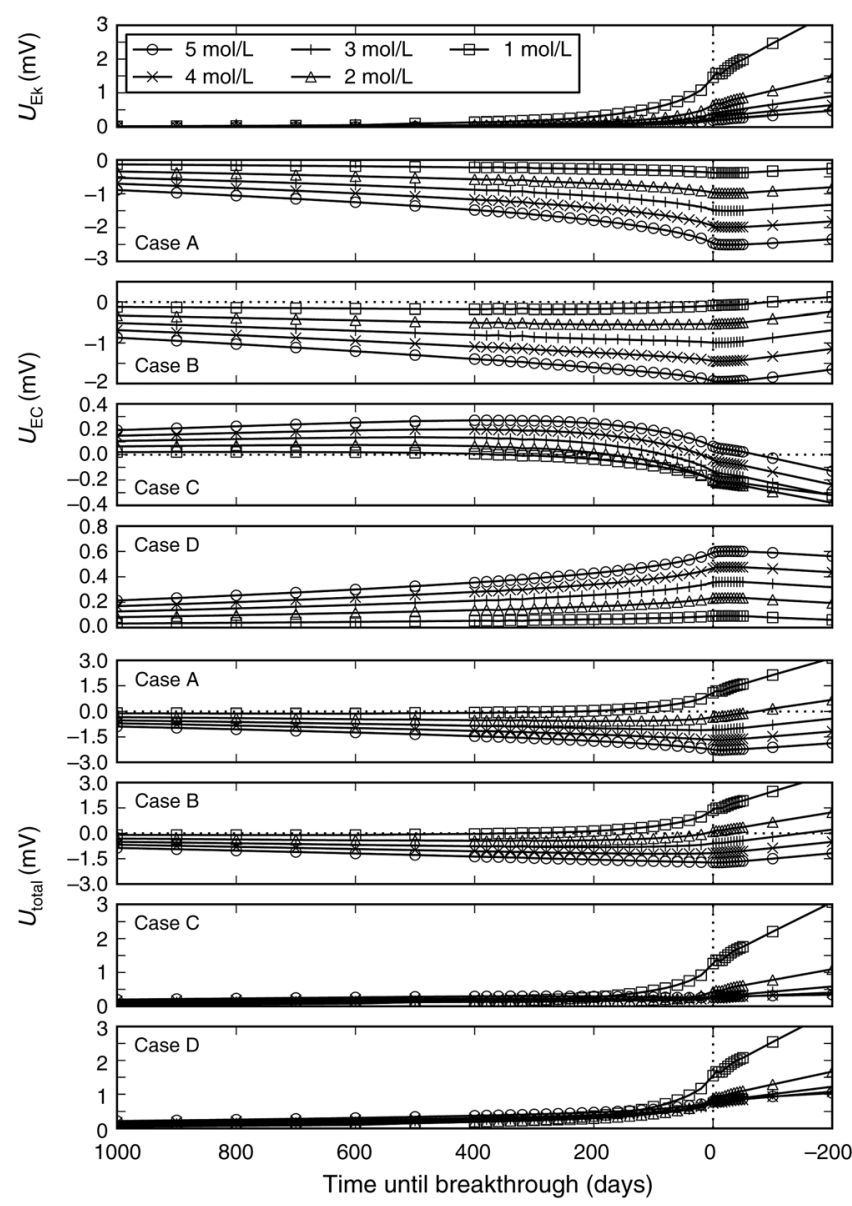

Figure 7. (Top to bottom) EK potential, EC potential for each case of $C_{r}$.EC and total SP for each case of $C_{r . \mathrm{EC}}$ as a function of time until water breakthrough occurs at the production well, at the center of the borehole, for different salinity formation brines. brine falls to $\lesssim 0.1 \mathrm{mV}$ just before water breakthrough. For cases $\mathrm{C}$ and D of the relative coupling coefficient, the SP signal measured at the production well is $\gtrsim 0.1 \mathrm{mV}$ for all salinities at least 600 days before breakthrough occurs. At low salinities ( $\sim 1 \mathrm{~mol} /$ liter $)$, the SP at the center of the borehole is dominated by the EK potential; at higher salinities $(\sim 5 \mathrm{~mol} / \mathrm{liter})$, the SP at the center of the borehole is dominated by the EC potential.

\section{Effect of injected brine salinity}

We finish by exploring the influence of the injected brine salinity upon the EK and EC components of the SP measured at a production well in a hydrocarbon reservoir. We simulate oil production by injecting different salinity brines at $30{ }^{\circ} \mathrm{C}$ into a reservoir containing 5 -mol/liter formation brine at $80^{\circ} \mathrm{C}$. Our results are presented in Figure 8, which shows the EK potential, the EC potential for each case of $C_{r, \mathrm{EC}}$, and the SP for each case of $C_{r, \mathrm{EC}}$ as a function of time until water breakthrough occurs at the production well, at the center of the borehole, for different salinity injected brines.

Altering the salinity of the injected brine by orders of magnitude does not cause the EK and EC potentials to change
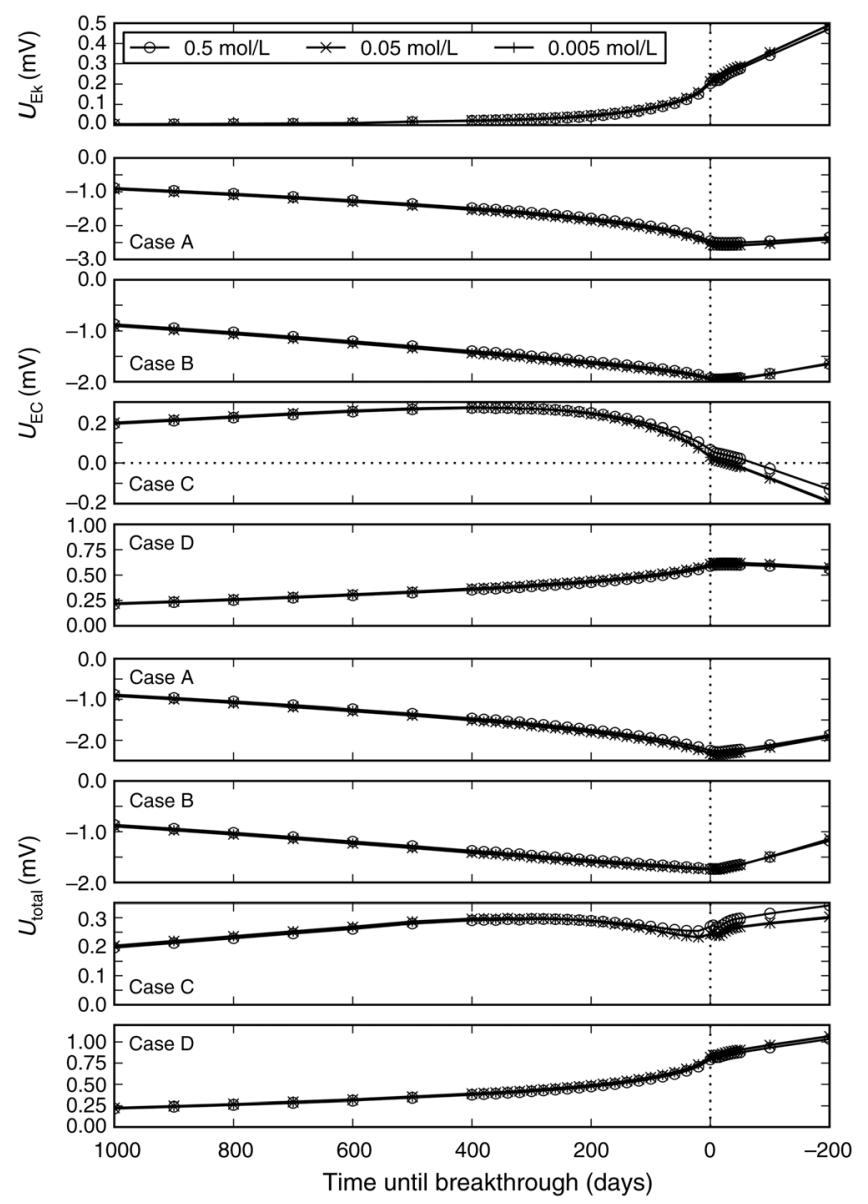

Figure 8. (Top to bottom) EK potential, EC potential for each case of $C_{r, \mathrm{EC}}$, and total SP for each case of $C_{r, \mathrm{EC}}$ as a function of time until water breakthrough occurs at the production well, at the center of the borehole, for different salinity injected brines. 
significantly. Similar results are obtained when injecting brine with 0.05 - and $0.005-\mathrm{mol} /$ liter salinities into a reservoir containing $0.5-\mathrm{mol} /$ liter formation brine (not shown). Consequently, the signal at the well is not sensitive to the salinity of the injected brine.

\section{CONCLUSIONS}

We have presented the results of comparing the EK, TE, and EC components of the SP signal generated in a hydrocarbon reservoir during production, by means of numerical simulation. The TE and EC coupling coefficients during multiphase flow are poorly understood, and we have considered four different cases. We investigated the effect of brine injected into reservoirs with different salinity formation brines as well as different-salinity brines injected into a reservoir with a fixed, more saline formation brine.

The injection of $0.5-\mathrm{mol} /$ liter brine at $30^{\circ} \mathrm{C}$ into a hydrocarbon reservoir containing 5-mol/liter formation brine at $80^{\circ} \mathrm{C}$ leads to an EK potential that is a maximum at the saturation front, increasing in magnitude to approximately $0.2 \mathrm{mV}$ as the front reaches the production well. Thermoelectric and EC potential signals are also generated. The TE potential is relatively large in magnitude in the reservoir $(\sim 20 \mathrm{mV})$ but small $(\lesssim 0.1 \mathrm{mV})$ at the production well until long after water breakthrough occurs as a result of the lag in the temperature front relative to the saturation front. The EC potential is also relatively large in magnitude in the reservoir $(\sim 15 \mathrm{mV})$, and the salinity front more closely follows the saturation front; consequently, the magnitude of the EC potential $(\gtrsim 0.2 \mathrm{mV})$ is noticeable at the well some time $(\sim 600$ days $)$ before water breakthrough occurs.

For three of the four cases of the TE and EC relative coupling coefficients we considered, we found that the EC component of the SP contributes at least $60 \%$ to the total signal measured at the production well. When the hydrocarbon reservoir acts as an uncharged porous medium ahead of the saturation front and a perfect membrane behind the front (case $\mathrm{C}$ of the TE and EC relative coupling coefficients), the $\mathrm{EK}$ component of the SP begins to contribute more than the TE or EC components at the well approximately 200 days before water breakthrough occurs. However, in this case, the SP signal at the production well relative to the SP signal 1000 days before breakthrough is always less than $0.1 \mathrm{mV}$, which suggests it would not be observable above background noise.

Varying the salinities of the formation and injected brines in our simulations, we find that the EC potential depends on how the coupling coefficient is modeled ahead of the saturation front and contributes more to the overall SP than the EK potential at high salinity ( $\sim 5 \mathrm{~mol} /$ liter $)$; however, at low salinity $(\sim 1 \mathrm{~mol}$ /liter), the EK potential contributes more than the EC potential.

Our results suggest that EK and EC effects need to be taken into account when interpreting SP signals in hydrocarbon reservoirs, but TE effects may be ignored in typical production scenarios. Moreover, for highly saline or relatively fresh formation brine, the overall SP signal may be used to detect an advancing waterfront at a production well and subsequently predict water breakthrough. These results are promising from the point of view of monitoring and controlling oil production in hydrocarbon reservoirs by means of water flooding. However, the coupling coefficients used in this study, and particularly the relative coupling coefficients, are uncertain because of a lack of available data. Further research is necessary to determine the nature of these parameters.

\section{ACKNOWLEDGMENTS}

We are grateful to Shell International Exploration and Production B.V. for funding this study. We would also like to thank Evert C. Slob, Mark Everett, and three anonymous reviewers for their comments and suggestions, which greatly improved this paper.

\section{REFERENCES}

Anderson, W. G., 1986, Wettability literature survey — Part 3: The effects of wettability on the electrical properties of porous media: Journal of Petroleum Technology, 38, 1371-1378, doi:10.2118/13934-PA.

- 1987, Wettability literature survey — Part 5: The effects of wettability on relative permeability: Journal of Petroleum Technology, 39, 1453-1468.

Bear, J., 1972, Dynamics of fluids in porous media: Dover Publ. Inc.

Braun, B. M., and H. Weingartner, 1985, Transference numbers of aqueous $\mathrm{NaCl}$ and $\mathrm{Na}_{2} \mathrm{SO}_{4}$ at $25^{\circ} \mathrm{C}$ from EMF measurements with sodiumselective glass electrodes: Journal of Solution Chemistry, 14, no. 9, $675-686$.

Chen, M.-Y., B. Raghuraman, I. D. Bryant, and M. Supp, 2006, Streaming potential applications in oil fields: Annual Technical Conference and Exhibition, Society of Petroleum Engineers, SPE 102106.

Corwin, R. F., and D. B. Hoover, 1979, The self-potential method in geothermal exploration: Geophysics, 44, 226-245, doi:10.1190/1.1440964.

Dake, L. P., 1978, Fundamentals of reservoir engineering: Elsevier Science Publ. Co., Inc.

Darnet, M., A. Maineult, and G. Marquis, 2004, On the origins of self-potential (SP) anomalies induced by water injections into geothermal reservoirs: Geophysical Research Letters, 31, L19609, doi:10.1029/2004GL020922.

Fitterman, D. V., 1978, Electrokinetic and magnetic anomalies associated with dilatant regions in a layered earth: Journal of Geophysical Research, 83, no. B12, 5923-5928, doi:10.1029/JB083iB12p05923.

Freedman, R., and B. E. Ausburn, 1985, The Waxman-Smits equation for shaly sands: I. Simple methods of solution; II. Error analysis: The Log Analyst, 26, 11-24.

Hassanizadeh, M., and W. G. Gray, 1993, Thermodynamic basis of capillary pressure in porous media: Water Resources Research, 29, 3389 3405, doi:10.1029/93WR01495.

Hunter, R. J., 1981, Zeta potential in colloid science: Academic Press.

Jaafar, M. Z., J. Vinogradov, and M. D. Jackson, 2009a, Measurement of streaming potential coupling coefficient in sandstones saturated with high salinity $\mathrm{NaCl}$ brine: Geophysical Research Letters, 36, L21306, doi:10.1029/2009GL040549.

Jaafar, M. Z., J. Vinogradov, M. D. Jackson, J. H. Saunders, and C. C. Pain, 2009b, Measurements of streaming potential for downhole monitoring in intelligent wells: Middle East Oil and Gas Show and Conference, Society of Petroleum Engineers, 120460-MS

Jackson, M. D., 2008, Characterization of multiphase electrokinetic coupling using a bundle of capillary tubes model: Journal of Geophysical Research, 113, B04201, doi:10.1029/2007JB005490.

, 2010, Multiphase electrokinetic coupling: Insights into the impact of fluid and charge distribution at the pore scale from a bundle of capillary tubes model: Journal of Geophysical Research, 115, B07206, doi:10.1029/2009JB007092.

Leinov, E., J. Vinogradov, and M. D. Jackson, 2010, Salinity dependence of the thermoelectric coupling coefficient in brine-saturated sandstones: Geophysical Research Letters, 37, L23308, doi:10.1029/2010GL045379.

Lynch, E. J., 1962, Formation evaluation: Harper \& Row Publishers, Inc.

Maineult, A., M. Darnet, and G. Marquis, 2006, Correction to "On the origins of self-potential (SP) anomalies induced by water injections into geothermal reservoirs": Geophysical Research Letters, 33, L20319, doi:10.1029/2006GL028211.

Marshall, D. J., and T. R. Madden, 1959, Induced polarization, a study of its cases: Geophysics, 24, 790-816, doi:10.1190/1.1438659.

Ortiz, J. I., J. S. Osoba, and W. D. von Gonten, 1973, Relationship of the electrochemical potential of porous media with hydrocarbon saturation: The Log Analyst, 14, 25-32.

Revil, A., 1999, Ionic diffusivity, electrical conductivity, membrane and thermoelectric potentials in colloids and granular porous media: A 
unified model: Journal of Colloid and Interface Science, 212, no. 2, 503-522, doi:10.1006/jcis.1998.6077.

Saunders, J. H., M. D. Jackson, and C. C. Pain, 2006, A new numerical model of electrokinetic potential response during hydrocarbon recovery: Geophysical Research Letters, 33, L15316, doi:10.1029/2006GL026835.

2008, Fluid flow monitoring in oil fields using downhole measurements of electrokinetic potential: Geophysics, 73, no. 5, E165-E180, doi: $10.1190 / 1.2959139$.

Schlumberger, 2009, ECLIPSE technical description 2009.1: Schlumberger

Telford, W. M., L. P. Geldart, and R. E. Sheriff, 1990, Applied geophysics, 2nd ed.: Cambridge University Press.
Vinogradov, J., M. Z. Jaafar, and M. D. Jackson, 2010, Measurement of streaming potential coupling coefficient in sandstones saturated with natural and artificial brines at high salinity: Journal of Geophysical Research, 115, B12204, doi:10.1029/2010JB007593.

Worthington, A. E., J. H. Hedges, and N. Pallat, 1990, SCA guidelines for sample preparation and porosity measurement of electrical resistivity samples: Part I - Guidelines for preparation of brine and determination of brine resistivity for use in electrical resistivity measurements: The Log Analyst, 31, 20-28.

Wyllie, M. R. J., 1951, An investigation of the electrokinetic component of the SP curve: AIME Transactions (American institute of Mining, Metallurgical, and Petroleum Engineers), 192, 1-11. 